\title{
The Quantum Hall Effect: Helicity, Graphite and Graphene
}

\author{
Keshav N. Shrivastava, Senior Member, IACSIT
}

\begin{abstract}
The Hall resistivity is explained to arise from the spin and angular momemtum which leads to fractional charge in a natural way. There are helical orbits for the electrons in a magnetic field so that the + sign in spin gives one helicity while the - sign gives another, which is also related to the sign of the electron velocity. The fractions which arise in the graphite resistivity are explained in terms of principal fractions, resonances, sum processes and clusters. The graphene Hall effect data is explained by using the Landau levels with special treatment of spin. The two layer graphene is well explained. A small number of fractions are explained in terms of sample-dependent clusters which are found in some of the samples. While most of the fractions are a property of pure samples, some of the fractions are induced by the clusters.
\end{abstract}

Index Terms-Graphene, graphite, helicity, quantum hall effect, special spin.

\section{INTRODUCTION}

Recently, we have described the correct theory of the quantum Hall effect as well as explained the origin of 101 fractions found in the experimental data [1]. The cyclotron frequency which appears in the Landau levels is replaced by double valued helical values. The Lande's formula is corrected to include the time-reversed conjugate states. Only the $l=0$ states are used in the original Landau theory which requires to be modified for finite $l$ value. Since $\mathrm{j}=l \pm \mathrm{s}$ is the total angular momentum keeping only $l=0$ leaves out the important effects at high fields. The energy levels which result by these corrections lead to the fractions in the flux-quantized resistivity measured in the Hall effect. The concept of helicity is introduced to understand the right and left moving electrons. Usually the velocity is single valued except that in complex clusters there are rotations which allow two signs of the velocity. Hence, as long as charge is conserved particles can occur with reversed helicity. In a single particle this will not happen but in a cluster it is possible.

In this paper, we describe the concept of helicity as applied to the quantum Hall effect of electrons in a high magnetic field and low temperatures and also explain the experimental data of fractions in the Hall effect of graphite and graphene. We look for the evidence of the mixed helicity as well as "reversed helicity". We analyze the experimental data of the quantum Hall effect measurements of noise power to look for the effect of helicity. We find that noise power depends on the helicity. It is possible to heat the samples by a heater wire and monitor the motion of the particles. Hence we explain that while one particle moves to the right, the conjugate will

Manuscript received October 1, 2012; revised December 5, 2012.

K. N. Shrivastava is with the University of Hyderabad, India. (e-mail: keshav1001@yahoo.com). move to the left. This motion of the particles is controlled by the helicity. Hence all particles occur in pairs. The original observation of the quantum Hall effect by von Klitzing et al [2] and Tsui et al. [3] had no theory and the efforts of Laughlin [4] did not yield an interpretation of the data and comparisons with the one-component plasma did not find the ground state. It was thought that the Coulomb correlations may lead to a fractional charge of the electron but this theory [5] does not depend on spin. The experimental data is consistent with the fractional charge.

\section{HELICITY}

\section{A. Spin and Helicity}

The helicity of a particle is defined as the sign of $\boldsymbol{\sigma} \cdot \mathbf{p} /|\mathbf{p}|$ where $\sigma$ is the spin without the factor of $1 / 2$ and $\mathbf{p}$ is the linear momentum. The photon is a boson with two polarizations, one along the direction of motion and the other opposite to it. When light is incident on a tilted plane, there is an electric field perpendicular to that plane and when the helicity is changed the direction of the electric field reverses. The helicity of the photon for circular polarization is defined as $\sin 2 \varphi$ so that it becomes a continuous variable with zero value at a few points. The photocurrent normal to the plane is proportional to the helicity [6]. In the case of particles, there are only two values of the helicity, + and -. This means that the helicity of the photon reverses but in the case of particles, the "reversed helicity" is not found except in the case of Majorana particles. In the quantum Hall effect the resistivity shows plateaux at a fraction of $\mathrm{h} / \mathrm{e}^{2}$ so that the fractional charge of a particle can be measured.

\section{B. Spin and Helicity in Resistivity}

In single particles, the helicity is defined as the projection of its spin along the direction of motion. It is impossible to change the helicity of a particle because it would then become its antiparticle. In the solid state it is possible to consider a cluster of particles which permits the observation of a phenomenon not found in single particles. The wave function of a particle may be made of a linear combination of two particles which differ in helicity. The helicity of one particle may be positive while that of the other may be negative and we can make a third particle which will be a combination of the first and the second particle. A particle of mixed helicity means that it is made of particles of different helicities. It does not mean that a particle of positive helicity can change itself into that of negative helicity. This means that "reversed helicity" does not occur. Under the rotation it is possible to change the direction of the velocity and hence the helicity of a particle in a molecule or in a solid but the charge has to be conserved. In the quantum Hall effect the 
resistivity is quantized at the plateaux. The usual Hall effect occurs with the resistivity linearly proportional to the magnetic field,

$$
\rho_{x y}=\frac{B}{n e c}
$$

where $B$ is the magnetic induction, e is the electron charge, $c$ is the velocity of light and $n$ is the electron concentration, the number of electrons per unit area for the slab of a metal. The flux quantization leads to plateaux at which,

$$
\rho_{x y}=\frac{h}{i e^{2}}
$$

where $h$ is the Planck's constant and $i$ is an integer. When angular momentum is considered, $i$ becomes a fraction. Due to particle-antiparticle symmetry the fractions occur in pairs, one for the positive spin and the other for the negative spin. For single particles, the resistivity at the plateau is given by,

$$
\rho_{x y}=\frac{h}{\frac{1}{2} g_{ \pm} e^{2}}
$$

where $g=(2 j+1) /(2 l+1)$ and $j=l \pm s$. The \pm sign in the expression for $\mathrm{j}$ introduces the particle-antiparticle symmetry. Since the signs occur in the spin, the values of the resistivity corresponds to two helicities. The values of $(1 / 2) g_{ \pm}$for the two helicities give two different plateaux in the resistivity. The spin as well as the helicities thus enter in the problem of Hall resistivity. The fractions which occur for a single value of the $l$ and $s$ are called "principal fractions". The two-particle states occur for $v_{1}+v_{2}$ where $v=(1 / 2) g_{ \pm}$and the effective charge of a particle becomes $e^{*}=(1 / 2) g e$. Similarly, the resonances occur at $v_{1}-v_{2}$. In $A l_{x} G a_{1-x} A s / G a A s$ heterostructures, clusters of electrons are often formed in which case the spin is different from $1 / 2$. The large values of spin are required to understand the plateaux which depend on the sample and show spin wave reduction in the value of the spin. The spin larger than $1 / 2$ occurs in clusters which are subject to rotations. Hence "roton" type effects are seen in the quantum Hall effect of clusters of electrons.

\section{Two-Particle States}

The single-particle states with spin $1 / 2$ occur at,

$$
\frac{1}{2} g_{ \pm}=\frac{l+\frac{1}{2} \pm s}{2 l+1}
$$

The positive sign corresponds to + helicity (right handed) and the negative sign corresponds to - helicity (left handed). For $l=0$ the above expression reduces to $(1 / 2) \pm s$ so that the effective charge for $s=1 / 2$ becomes 1 and corresponds to + helicity and the negative sign gives zero charge and corresponds to - helicity. For $l=1$, the denominator is $2 l+1=3$ and the numerator gives $(3 / 2) \pm s$ which is 2 for + and 1 for the negative sign. Hence $1 / 3$ belongs to negative helicity and $2 / 3$ to positive helicity. These are single particle states which can not rotate. There is no doubt that all of the predicted series of fractions $l /(2 l+1)$ as well as $(l+1) /(2 l+1)$ are the same as in the experimental data. The two-particle state of charge $1 / 3$ occurs at $(1 / 3)+(1 / 3)=2 / 3$ with negative helicity. The one particle state at $2 / 3$ has positive helicity. Hence the two-particle state at $2 / 3$ is degenerate with the one particle state at $2 / 3$ so that $2 / 3$ has mixed helicity. The two particle state of $2 / 3$ occurs at $4 / 3$ which will be of mixed helicity. Similarly, $5 / 3$ made from $(1 / 3)+(4 / 3)=5 / 3$ belongs to mixed helicity. By rotation the direction of velocity changes, and the sign of $\mathrm{s}$ changes so that $(2 / 3)+(2 / 3)+(1 / 3)=(2 / 3)+(1 / 3)+(2 / 3)=5 / 3$ is an example of reversed helicity with conserved charge. We have started with the odd number of particles and reversed the helicities of only two values. It is possible to start with even number of values in which we reverse the helicities of even number of charges so that we reverse the helicities without loosing the charge conservation. The state $7 / 3$ is made of $(2 / 3)+(2 / 3)+(1 / 3)+(2 / 3)=7 / 3$. This is a cluster of four electrons. Upon rotation $1 / 3$ changes into $2 / 3$ and $2 / 3$ changes into $1 / 3$ so that $7 / 3$ is an example of mixed helicities as well as reversed helicities. Similarly $8 / 3=1 / 3+7 / 3$ is an example of mixed as well as "reversed helicities".

\section{Noise Power and Helicity}

In the band theory of solids, the sum of the electron charge and the hole charge is zero. However, when a hole is created, an electron must be removed from the same position. Hence, the annihilated eigen value is $f$ when created eigen value is (1-f). The sum of the two values is 1 . If charge is conserved, the fractional charge $1 / 3$ requires that a charge of $2 / 3$ should also arise. In the flux quantization the charge is e because flux is quantized as n'hc/e. Hence the charge is not e but it is $\mathrm{e} / \mathrm{n}$ '. Hence the charge can become e/2 and not necessarily e. The sum of the charges $1 / 3+2 / 3=1$ can also become $(1 / 2)[1 / 3+2 / 3]=1 / 2$. In the periodic potential the $1 / 3$ is replaced by $\pm 1 / 3$ and $2 / 3$ is replaced by $\pm 2 / 3$ and so on and so forth. For electronic states of fractional charges such as $2 / 3,3 / 5,8 / 3$, $5 / 2$ etc., there are hole states of opposite charge. The noise power at a point is of the form,

$$
S=2 g_{Q} \int_{o}^{\infty} f(1-f) d E
$$

where $f(1-f)$ is a fermion factor which arises from the creation of an electron at one site accompanied with the destruction of an electron at another site and $g_{Q}$ is the conductance of the material at the quantum point contact. The thermal excess noise power is of the form,

$$
S_{\text {excess }}=2 \Delta \varepsilon_{F} g_{Q} t(1-t)\left[\operatorname{coth} \frac{\Delta \varepsilon_{F}}{2 k_{B} T}+\frac{2 k_{B} T}{\Delta \varepsilon_{F}}\right]
$$

where $\Delta \varepsilon_{F}$ is the change in the Fermi energy, $t(1-t)$ is the transmission probability and $T$ is the temperature. The conductance at a point at $1 / 3$ is for example,

$$
g_{Q}=\frac{\frac{1}{3} e^{2}}{h}
$$


For $l=0, s=+1 / 2,(1 / 2) g=(1 / 2)-s=0$ which is electrically neutral or a neutral particle. Two particles of charge $1 / 3$ each and one hole of charge $-2 / 3$ create a zero charge. Similarly, two holes of charge $-1 / 3$ each and one particle of charge $2 / 3$ can produce charge neutrality. In the experimental data [7] of $A l G a A s / G a A s$ also there is a zero energy state with $\mathrm{g}=0$. The excess noise power has the factor $g_{\mathrm{Q}}=(1 / 3) e^{2} / h$ accompanied with the factor $(2 / 3) e^{2} / h$ which differ in helicity. Hence the noise power changes upon change in helicity. Some times the experimentalists [8] use the idea of chirality which is similar to that of helicity defined in the present work as the sign of p.s. The velocity of the electron appears in the helicity so that upstream particles have one helicity while the down stream have another helicity. Hence $1 / 3$ and $2 / 3$ travel in opposite directions. It is possible to put a heater wire in the sample to see the direction of motion of the fractionally charged particles [9]-[10].

\section{GRAPHITE DATA}

According to our theory, there are quasiparticles of fractional as well as integer charges and the spin and charge are coupled. Hence, a modified Bohr magneton emerges and resistivity depends on the spin. There are fundamental charges given by $e^{*} / e=(1 / 2) g$. where $g=(2 j+1) /(2 l+1)$ so that the resistivity becomes $\rho=h /\left[(1 / 2) g e^{2}\right]$. In heterostructures, the spin need not be $1 / 2$ because there is cluster formation. For example, the spin of a cluster may be $3 / 2$ or $5 / 2$, etc. There are two particle states so that $\omega_{1}+\omega_{2}$ is possible. Similarly, there are resonances so that $\omega_{1}-\omega_{2}$ is also allowed. Hence, quasiparticle charge is determined from (i) spin-charge coupling, (ii) two-particle states, (iii) resonances and (iv) electron clustering. We explain the fractional charges found in graphite. The experimental measurements have been performed by Kopelevich et al. [11] so that we obtain the fractions from their work which are, 2/7, 1/4, 2/9, $1 / 5,2 / 11,1 / 6,2 / 15,1 / 8,2 / 17$ and $1 / 9$. The energy of a state is given by $(1 / 2) g(n+1 / 2)$ so that we consider two oscillators with energies, $E_{1}=(1 / 2) g_{1}\left(n_{1}+1 / 2\right)$ and $E_{2}=(1 / 2) g_{2}\left(n_{2}+1 / 2\right)$. The energy difference between these states is $(1 / 2) g\left(n_{1}-n_{2}\right)$. For $l=3, \quad 2 l+1=7$ and for positive sign in $(1 / 2) g=[l+(1 / 2) \pm s] /(2 l+1)=4 / 7, \quad(1 / 2) g_{1} n_{1}-(1 / 2) g_{2} n_{2}$ $+(1 / 2) g_{1}(1 / 2)-(1 / 2) g_{2}(1 / 2)=(1 / 2)(1 / 2) g_{1}$ for $n_{1}=n_{2}=0$, $(1 / 2) \mathrm{g}_{2}=0$ for the second oscillator which has $l=0$, -ve sign and $s=1 / 2$ so that $(1 / 2) g_{2}=0$. Hence, $(1 / 2)(1 / 2) g_{1}=2 / 7$. The ingredients we put are two oscillators with different values of $l$ and $s$ which are the orbital and spin angular momenta quantum numbers. The effective charge which depends on spin also determines the resistivity. Hence the resistivity depends on spin. In the electron clusters, spin can become zero, so that we put $s=0$ to obtain $(1 / 2) g=$ $[l+(1 / 2)] /(2 l+1)=1 / 2$ or $g=1$. In the formula $(1 / 2)[(1 / 2) g-0]$ for $g=1$ we obtain $1 / 4$. For $l=4,2 l+1=9$ and for $s=1 / 2$ for negative sign, we obtain $(1 / 2) g=4 / 9$. In the expression, $(1 / 2)\left[(1 / 2) g_{1}-(1 / 2) g_{2}\right]$ we have $g_{2}=0$ and $(1 / 2) g_{1}=4 / 9$ so that the effective charge becomes $(1 / 2)(1 / 2) g_{1}=2 / 9$. For $l=2$, we have $2 l+1=5$ and $l / 2 l+1=2 / 5$ and $(l+1) /(2 l+1)=3 / 5$. We calculate the resonance state at $(1 / 2) g\left[n_{1}+(1 / 2)\right]-(1 / 2) g\left[n_{2}+(1 / 2)\right]$ at $(1 / 2) g\left(n_{1}-n_{2}\right)$ which comes at $3 / 5-2 / 5=1 / 5$. For $l=5$ we have $l / 2 l+1=5 / 11$ and $(l+1) /(2 l+1)=6 / 11$. The value of $(1 / 2)(6 / 11-0)=3 / 11$. The resonance state now occurs at $5 / 11-3 / 11=2 / 11$. Let us look at the flux quantization at $n^{\prime}$ hc/e so that for $n^{\prime}=2$, the charge is $\mathrm{e} / 2$. Hence for $n^{\prime}=2$, the effective value of $1 / 3$ changes to $1 / 6$. The original value for $l=1,2 l+1=3$ for negative sign is $1 / 3$. For $l=7$, the two series, $l / 2 l+1=7 / 15$ and $(l+1) /(2 l+1)=8 / 15$, $(1 / 2)(1 / 2) g=4 / 15$ and for $n^{\prime}=2,4 / 15$ becomes $2 / 15$. We have already obtained $1 / 4$ which for $n^{\prime}=2$ becomes $1 / 8$. For $l=8$, $2 l+1=17$ and the principal fractions are $l / 2 l+1=8 / 17$ and $9 / 17$. We have $(1 / 2)(1 / 2) g=4 / 17$ which for $n '=2$ gives $2 / 17$. For $l=4$, $l /(2 l+1)=4 / 9$ and $(l+1) /(2 l+1)=5 / 9$. The resonance state of these two occurs at $5 / 9-4 / 9=1 / 9$. This explains all of the fractions observed in the fractional quantum Hall effect of graphite. This confirms that our theory provides the correct interpretation of the quantum Hall effect data.

\section{GRAPHENE}

The band structure of graphite was calculated by Wallace [12] by using "tight binding" approximation. In this calculation, the valence and the conduction bands do cross at a point which can be visualized as a double cone. The upper cone is the conduction band and the lower cone is the valence band. The two cones may be of equal angle. If the cone angles are equal, then there are symmetries in the energy. However, if the two cone angles are not equal, the energies will not be symmetric. In the symmetric case, the energies of the quasiparticles in the two bands are equal in the magnitude and can be represent by $\pm \mathrm{E}$. Then there is analogy with the relativistic expression, $\pm\left(c^{2} p^{2}+m^{2} c^{4}\right)^{1 / 2}$. The energies of the quasiparticles can be mapped to the relativistic expression. Therefore, the band structure of graphene has been recalculated by Latil and Henrard [13] who find that the number of layers is an important parameter in the problem. If the valence and conduction cones are symmetric, it is possible to describe the quasiparticles by the Dirac equation in which the velocity of light is replaced by the Fermi velocity. The point at which the two cones meet is called the "Dirac point", which has been considered by Partoens and Peeters [14]. In this way, the band structure has been obtained from the Schrödinger as well as from the Dirac theory. In the non-relativistic theory the symmetric energy levels can be obtained from the Kramers conjugate states such as $\pm 1 / 2$ for the spin angular momentum. The bands can be distorted by the impurities [15]. Under these conditions, some energy levels have been drawn on the basis of relativistic theory and some on the basis of non-relativistic theory. Therefore, it is important to know the relativistic results as distinguished from the non-relativistic theory.

We obtain the band structure of several layers of graphene and for a variety of stacking of layers by using non-relativistic density-functional theory [16]. We discuss the gap energies as a function of number of layers. We discuss the symmetries near the crossing points. Our calculations are based on non-relativistic Schrödinger equation, and hence relativistic effects have not been obtained. Some of the crossing points actually have a small gap so these should be called "Schrödinger points". The velocity of light can not be replaced by the particle velocity. 
While linearization of the dispersion may be an interesting problem, it is not immediately needed for the interpretation of the experimental data. Hence we have not done the linearization of crossing of bands.

DMol3 Band Structure

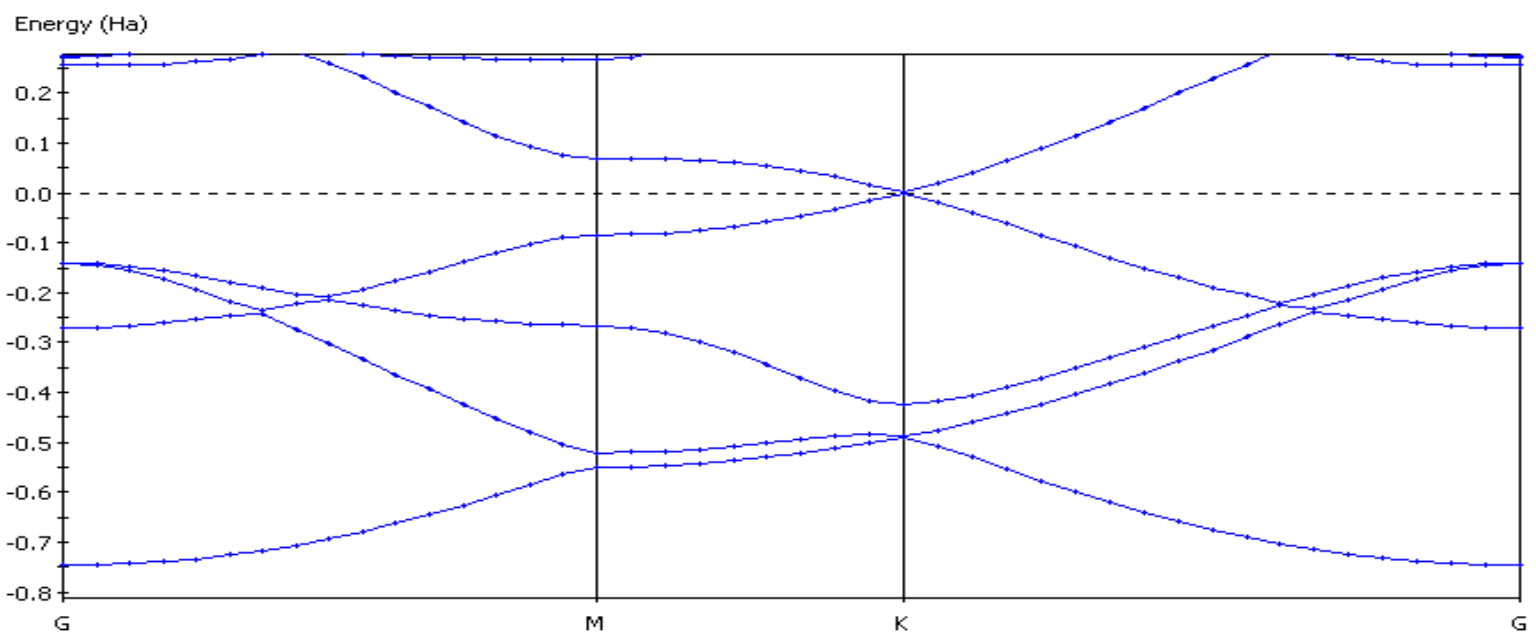

Fig. 1. The band structure of a single layer of graphene.

DMol3 Band Structure

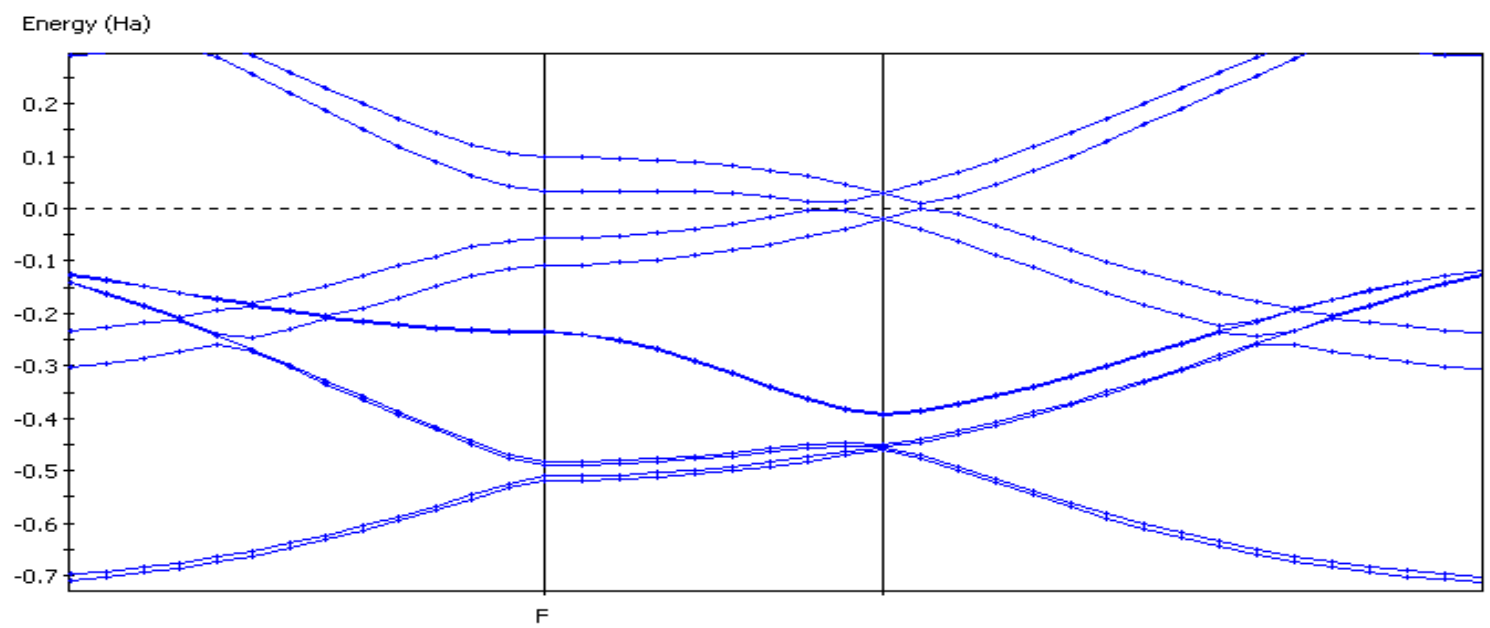

Fig. 2. The band structure of AA layers

1) First of all we use the density functional theory to optimize the bond lengths and angles for which the energy of the Schrödinger equation is a minimum. For the configuration of the atoms for which the energy is minimized, we calculated the band structure at several points in the $\mathrm{k}$ space. For the points $\mathrm{G}(0,0,0), \mathrm{M}(0,0.5,0), \mathrm{K}(-0.333$, $0.667,0)$ for a single layer of graphene, the band structure is shown in Fig. 1.

2) At the point $K$, there is an apparent crossing. If we see this point carefully, it is not a crossing point and there is a gap of $27.212 \mathrm{meV}$. At the point M, starting from zero energy the positive root is not equal to the negative root and hence the energies are not symmetric with respect to the zero value. At the point $\mathrm{G}$ also, the positive and negative roots are not equal and hence the results are non-relativistic.

3) When we put a second layer of graphene on top of the first layer such that the hexagons of the second layer are on the top of those of the first, which we call the AA type, we obtain the band structure. In this case a close observation shows that the positive roots are not equal to the negative roots. The $\mathrm{F}(0,0.5,0)$ point clearly shows four roots, two above zero and two below zero but the magnitudes of energies are not symmetric about the zero as seen in Fig. 2.

4) In the three layers, the AAA type, the bands show unusual bending without crossing. The band from the top comes down and bends back. Similarly, the lower band comes up and bends back without crossing. A third band comes down and comes all the way down through the space created by the first two bands but there is no crossing.

5) In the four layers, AAAA type, there is no crossing and the positive and the negative energies are not equal in magnitude. In the five layers of AAAAA tpe, the bands become degenerate but the positive and the negative roots are not equal. In six layers of AAAAAA type there is a large gap and the positive and negative roots are not equal in magnitude. In seven layers of the type AAAAAAA, the 
bands become degenerate but not symmetric with respect to zero value. In eight layers of all A type there is a degenerate point but not symmetry point. In nine layers also there is a degeneracy point but not symmetry point. In ten layers, there is a crossing point which has degenerate solutions also for some wave vectors but there is no symmetry about the zero energy. The results for eleven and twelve layers are also of this type. Hence, it can be said that there are many bands which may cross or become degenerate but not symmetric about zero energy.

6) The centre of the hexagon of A layer does not have any atom. In the second layer we arrange the hexagons in such a way that an atom comes below the centre of the hexagon of the first layer. Therefore, there is an atom in the B layer just below the centre of the hexagon of A layer. In this way, we can stack layers of the hexagons with alternate A and B type layers. The gap energy (number of layers) are $4.8 \mathrm{meV}$ (2), $0.33 \mathrm{eV}(3), 2.99 \mathrm{meV}$ (4), $13.6 \mathrm{meV}$ (5), $2.99 \mathrm{meV}$ (7), 5.71 meV (9), $3.8 \mathrm{meV}$ (11). The numbers 6, 8, 10 and 12 do not have a clear gap. The $\mathrm{AB}$ stack (number of layers=2) has a gap but the angle between the line of constant energy and that of the conduction band is not equal to that in the valence band at the points, $\mathrm{G}(0,0,0), \mathrm{F}(0,0.5,0), \mathrm{K}(-0.333,0.667,0)$ and $\mathrm{G}(0,0,0)$. In the case of twelve layers of alternate $A$ and B type the crossing is shown in Fig. 3. The symmetry and the bending of one of the bands are clearly seen. As the conduction band approaches the crossing point, there is a deflection in the energy as a function of wave vector.

7) In the $C$ type layer, one of the atoms is below the centre of the hexagon of A layer and the hexagon is. rotated till another atom of the $\mathrm{C}$ layer comes directly below one of the atoms of A layer. The stacks of the ABC type have been made. The points examined are $\Gamma(0,0,0), \mathrm{K}(0,0.5,0)$ and $\mathrm{K}^{\prime}(-0.333,0.667,0)$. The band gaps are as follows $\mathrm{ABC}$ $0.626 \mathrm{eV}, 0.299 \mathrm{eV}$; ABAC $24.49 \mathrm{meV}$, ABCA $0.61 \mathrm{meV}$; ABCAB $2.45 \mathrm{meV}, \mathrm{ABCABC} 0.38 \mathrm{eV}, 0.626 \mathrm{eV}, 0.299 \mathrm{eV}$; ABCABCA $1.224 \mathrm{meV}, \quad$ ABCABCAB $2.77 \mathrm{meV}$, $\begin{array}{llllll}A B C A B C A B C & 0.489 & \mathrm{eV} & 0.626 \mathrm{eV}, & 0.299 & \mathrm{eV} \text {; }\end{array}$ ABCABCABCA $1.361 \mathrm{meV}$, ABCABCABCAB $2.313 \mathrm{meV}$, ABCABCABCABC $0.381 \mathrm{eV}, 0.626 \mathrm{eV}, 0.229 \mathrm{eV}$. Thus in a variety of $\mathrm{ABC}$ type layers up to 12 layers there is no crossing.

8) We have made 12 layers of A type, 12 layers of $A B$ type and 12 layers of $\mathrm{ABC}$ type of graphene. The single layer has a small gap. Most of the stacks have a gap of the order of a few $\mathrm{meV}$. There are some cases of AB stacks, particularly, even number of stacks with number of layers equal to $6,8,10$, and 12 which do have crossing. The positive energies at the crossing points are not equal to negative energies in magnitude which is a quality of supersymmetry. In 48 different computations of band structure the “supersymmetry' is not found. All our calculations are based on Schrödinger non-relativistic theory. Zhang et al [17] represent the atoms by dumb bell of band structure in which the energies are the geometric average of the Fermi energy and the cyclotron frequency. The positive root is equal to the negative root. In the non-relativistic band structure, a supersymmetric band structure with equal values of positive and negative energies, is not found.

\section{DIRAC POINT}

The band structure of electrons which has carbon atoms should be non-relativistic, i.e., it has $\mathrm{p}^{2} / 2 \mathrm{~m}$ in the kinetic energy. The Dirac equation has linear $p$ because of the symmetry of the space and time. Hence, in the band structure crossing points, the dispersion is parabolic in the linear momentum and not linear. Near the crossing point, the energies are symmetric for the electron and the positron. Above the crossing point there are electrons and below the crossing points positrons do not occur in graphene. Replacing the velocity of light by the electron velocity (Fermi velocity) is not possible because of the special properties of the velocity of light. Above the crossing points the particles are negatively charged and hence we may assume that below the crossing point the particles are positively charged. We are plotting the energy, not the charge, but the "crossing point" may be thought to be electrically neutral or a neutral point. The Dirac Hamiltonian has only positively charged particles accompanied with the negatively charged particles but neutral particles do not occur. It will take quite a lot of work to introduce the neutral points. For the time being, the Dirac points do not occur in graphene and the reference to these points in journals is very ambitious. The replacement of the velocity of light by the Fermi velocity is unlikely to yield realistic results.

\section{GRAPHENE HALl EFFECT}

Usually the free electrons are in the $l=0$ state so that the electron gas models give a single value of the charge of the electron. In these experiments $l=0$ is the root cause of observing a single value for the electron charge. We consider (a) the finite value of $l$ and more spin symmetries than are usually taken into account. We allow two levels for $s=1 / 2$ and two more levels for spin $=-1 / 2$ so that there are four levels for spin $1 / 2$. These levels are not superimposed on each other because there are two separate $g$ values, one for spin $+1 / 2$ and the other for spin $-1 / 2$, which describes the quantized current correctly for $v=1$. The filling factor is, $v=\frac{1}{2} g_{ \pm}$which gives, one value for + sign and the other for - sign. We have thus introduced three different $g$ values, the usual $g$ value as well as $g_{ \pm}$. For $l=0$, we obtain $(1 / 2) g_{+}=1$ and $(1 / 2) g_{-}=0$, for $l=$ 1 , we get $(1 / 2) g_{+}=2 / 3$ and $(1 / 2) g_{-}=1 / 3$. The predicted values are the same as those measured. The effective charge is determined by the modification of the cyclotron frequency, $\hbar \omega_{\mathrm{c}}=\mathrm{g} \mu_{\mathrm{B}} \mathrm{B} \quad$ as, $\quad e_{\text {eff }}=(1 / 2) g_{ \pm} e=v e$. One of the series is $v=l /(2 l+1)$ and the other is $v_{+}=(l+1) /(2 l+1)$. These series exactly predict the observed series.

The formula can be rearranged as,

$$
\frac{1}{2} g_{ \pm}=\frac{l+\frac{1}{2} \pm s}{2 l+1}
$$

For $l=0$, the above formula gives, 


$$
\mathrm{e}^{* / \mathrm{e}}=\frac{1}{2} \pm s
$$

so that for $s=1 / 2$, the effective charge becomes zero or one. Both these charges are important because they are both experimentally observed. We introduce the concept of negative spin so that the number of energy levels is not limited by $2 S+1$. It does not matter much if this expression is relaxed. The number of levels will then be infinite and not $2 S+1$. Upto $2 \times 2$ matrix representation for the spin, the commutators are the same for the negative spin as for the positive spin. In fact, the levels resemble the harmonic oscillator. In the case of oscillations in a many-body system, we can remove the divergence in the energy by fixing the number of atoms but in the case of spins, the equally spaced levels can be made to continue upto $\infty$.

First of all, we write the infinite set of energy levels as follows.

$$
\frac{5}{2} g \mu_{B} H, \frac{3}{2} g \mu_{B} H, \frac{1}{2} g \mu_{B} H,-\frac{1}{2} g \mu_{B} H,-\frac{3}{2} g \mu_{B} H,-\frac{5}{2} g \mu_{B} H, \ldots
$$

which are solutions of the Hamiltonian, $\mathcal{H}=g \mu_{B} H_{z} S_{z}$. Here g is the splitting factor, $\mu_{B}$ is the Bohr magneton, $\mathrm{H}_{\mathrm{z}}$ the field along the $z$ direction and $S_{z}$ the z component of the spin. The above infinite series results for negative spin. For the positive spin, $1 / 2$, the levels are at $+(1 / 2) g \mu_{B} H$ and $-(1 / 2) g \mu_{B} H$. Next, we use both the positive as well as the negative sign in the total angular momentum so that $\mathrm{j}=l \pm \mathrm{s}$. In this case, we take the ratio,

$$
g=\frac{2 j+1}{2 l+1}
$$

Keeping both the signs in $\mathrm{S}$, the above can be written as,

$$
\mathrm{g}=\frac{2(l \pm s)+1}{2 l+1}
$$

For positive sign,

$$
\frac{1}{2} g_{+}=\frac{l+\frac{1}{2}+s}{2 l+1}
$$

and for negative sign,

$$
\frac{1}{2} g_{-}=\frac{l+\frac{1}{2}-s}{2 l+1}
$$

which are same as in (8). In the case of $l=0$, an interesting situation arises. The effective charge becomes related to spin,

$$
\mathrm{e} * / \mathrm{e}=\frac{1}{2} g_{+}=\frac{1}{2}+s \quad \text { (Spin-charge locking) }
$$

and

$$
\mathrm{e}^{*} / \mathrm{e}=\frac{1}{2} g_{-}=\frac{1}{2}-s \quad \text { (Spin-charge locking) }
$$

Since, the Bohr magneton, $\mu_{B}=e \hbar / 2 m c$ multiplies the $g_{ \pm}$ values, the effective charge of the electron can be written as $e^{*}=(1 / 2) g_{ \pm} e$. For positive $s=1 / 2$, the equation (15) gives $g_{+}=2$ and for negative sign we get $g_{-}=0$. The $g_{+}=2$ is the usual expected value but we also find, $g_{-}=0$, which gives zero energy. We substitute $g=g_{+}=2$ (for $s=1 / 2$ ) in the expression (10) to obtain the following energies,

$$
\mathrm{E}=5 \mu_{\mathrm{B}} \mathrm{H}, 3 \mu_{\mathrm{B}} \mathrm{H}, \mu_{\mathrm{B}} \mathrm{H},-\mu_{\mathrm{B}} \mathrm{H},-3 \mu_{\mathrm{B}} \mathrm{H},-5 \mu_{\mathrm{B}} \mathrm{H}, \ldots \text {, etc. }
$$

and from eq.(16) we get, $g=g=0(\mathrm{~s}=1 / 2)$ which substituted in (10) gives,

$$
\mathrm{E}=0 \text {. }
$$

This zero is very important because it is associated with zero charge. We can eliminate $\mu_{B} \mathrm{H}$ from the above by using,

$$
\mathrm{g} \frac{e \hbar}{2 m c} \mathrm{H}=\frac{g}{2} \hbar \omega_{c}=\hbar \omega_{c}
$$

for $g=2$. For $s=1$, from (15) $g_{+}=3$, so we substitute this value in (10) to obtain,

$$
\begin{aligned}
& \frac{15}{2} \mu_{\mathrm{B}} \mathrm{H}, \frac{9}{2} \mu_{\mathrm{B}} \mathrm{H}, \frac{3}{2} \mu_{\mathrm{B}} \mathrm{H},-\frac{3}{2} \mu_{\mathrm{B}} \mathrm{H},-\frac{9}{2} \mu_{\mathrm{B}} \mathrm{H}, \\
& -\frac{15}{2} \mu_{\mathrm{B}} \mathrm{H}, \ldots, \text { etc. }
\end{aligned}
$$

For $S=1$, with negative sign in front of spin, eq.(16) gives $\mathrm{g}=-1$. We substitute $g=g_{-}=-1$ in (10) to obtain,

$$
-\frac{5}{2} \mu_{\mathrm{B}} \mathrm{H},-\frac{3}{2} \mu_{\mathrm{B}} \mathrm{H},-\frac{1}{2} \mu_{\mathrm{B}} \mathrm{H},+\frac{1}{2} \mu_{\mathrm{B}} \mathrm{H},+\frac{3}{2} \mu_{\mathrm{B}} \mathrm{H},+\frac{5}{2} \mu_{\mathrm{B}} \mathrm{H}, \ldots,
$$
etc.

so that the positive and the negative energies get interchanged but there is no effect on the full energy level diagram. This is actually related to the invariance of the Hamiltonian with respect to the time reversal. For $s=3 / 2$, from eq.(15) $g_{+}=4$. We substitute $g=g_{+}=4$ in eq.(10) to obtain,

$$
10 \mu_{B} H, 6 \mu_{B} H, 2 \mu_{B} H,-2 \mu_{B} H,-6 \mu_{B} H,-10 \mu_{B} H, \ldots \text {, etc. }
$$

so that we obtain the series $, \pm 2, \pm 6, \pm 10, \pm 14, \pm 18$, etc. This series has the interval 4 . Not only the interval but the full series is the same as in the experimental data[18] at 9 Tesla. We have obtained the exact series without any approximation and all numbers are individually exact. Hence, the 
many-body corrections are not the cause of the series. For $s=3 / 2$, using the negative sign expression (16) we obtain, $(1 / 2) g=-1$. In this case, the energy levels of $g=-2$ are the same as that of $\mathrm{g}=2$ due to time reversal invariance. As the magnetic field is varied, different energy levels cross the Fermi level so that there are oscillations but that will not form plateaus. The plateaus are formed due to the flux quantization,

$$
\pi l_{\mathrm{o}}^{2} B=n \phi_{\mathrm{o}}
$$

where $\phi_{0}=h c l e$. We substitute the flux quantization condition in eq.(10) so that the magnetic field completely disappears,

$$
\begin{aligned}
& \frac{5}{2} \mathrm{~g} \mu_{\mathrm{B}}\left(\frac{n \phi_{o}}{\pi l_{o}^{2}}\right), \frac{3}{2} \mathrm{~g} \mu_{\mathrm{B}}\left(\frac{n \phi_{o}}{\pi l_{o}^{2}}\right), \frac{1}{2} \mathrm{~g} \mu_{\mathrm{B}}\left(\frac{n \phi_{o}}{\pi l_{o}^{2}}\right),-\frac{1}{2} \\
& \mathrm{~g} \mu_{\mathrm{B}}\left(\frac{n \phi_{o}}{\pi l_{o}^{2}}\right),-\frac{3}{2} \mathrm{~g} \mu_{\mathrm{B}}\left(\frac{n \phi_{o}}{\pi l_{o}^{2}}\right), \ldots,
\end{aligned}
$$

Not only that there are factors like, $5 / 2,3 / 2,1 / 2,-1 / 2,-3 / 2$, $-5 / 2, \ldots, \infty$, there is a factor of $n$ also which has come from the flux quantization. We substitute the flux quantization in the series (22) so that we find,

$10 \mu_{\mathrm{B}}\left(\frac{n \phi_{o}}{\pi l_{o}{ }^{2}}\right), 6 \mu_{\mathrm{B}}\left(\frac{n \phi_{o}}{\pi l_{o}{ }^{2}}\right), 2 \mu_{\mathrm{B}}\left(\frac{n \phi_{o}}{\pi l_{o}{ }^{2}}\right),-2 \mu_{\mathrm{B}}\left(\frac{n \phi_{o}}{\pi l_{o}{ }^{2}}\right),-6$

$\mu_{\mathrm{B}}\left(\frac{n \phi_{o}}{\pi l_{o}^{2}}\right),-10 \mu_{\mathrm{B}}\left(\frac{n \phi_{o}}{\pi l_{o}^{2}}\right)$

The series for $n=1$ is $\ldots, 10,6,2,-2,-6,-10, \ldots$ but for $n=2$ we have $\ldots, 20,12,4,-4,-12,-20, \ldots, \infty$. Now the interval is not 4 but 8 . The plateaus are now predicted at \pm 4 which was not a part of the first series. When the field is increased from 9 Tesla to 25 Tesla the plateaus at \pm 4 are indeed found. The remaining values are too weak. In this way we are able to predict the plateaus at \pm 4 in addition to those given by the series $2,6,10, \ldots$, etc. This means that our predicted values are in full agreement with the data. The values of 0 and 1 were already present in ref.[1]. Away from the plateau the field dependence is of the form of, $10 \mu_{B}\left(\frac{n \phi_{o}}{\pi l_{o}^{2}}\right)+10 \mu_{B} H$.

Large charges are found in the quantum Hall effect of graphene. For spin $3 / 2, l=0$, we get,

$$
\frac{1}{2} g_{+}=2
$$

or $g_{+}=4$. The harmonic oscillator type states are,

$$
n+\frac{1}{2}=\frac{1}{2}, 3 / 2,5 / 2,7 / 2,
$$

multiplying these by 2 due to (26),

$$
\left(n+\frac{1}{2}\right) 2=1,3,5,7, \ldots
$$

Making the two-particle states gives,

$$
\left(n+\frac{1}{2}\right) g_{+}=2,6,10,14,18, \ldots
$$

In the two layer system, we expect,

$$
\left(n_{1}+\frac{1}{2}\right) \frac{1}{2} g_{+}-\left(n_{2}+\frac{1}{2}\right) \frac{1}{2} g_{+}=\left(n_{1}-n_{2}\right) \frac{1}{2} g_{+}
$$

which is $n(1 / 2) \mathrm{g}$ with $g=4$, i.e., $2 n=2,4,6,8, \ldots$, and the two particle states are at $4,8,12,16, \ldots$ These predicted series are exactly the same as in the experimental data [18]. However, there is an alternative interpretation so that these are not the two particle states but they are four-particle states. In the eq.(30) the difference is shown. Let us use the sum instead of the difference so that the sign is changed to + instead of -, the result being,

$$
\left(n_{1}+\frac{1}{2}\right) \frac{1}{2} g_{ \pm}+\left(n_{2}+\frac{1}{2}\right) \frac{1}{2} g_{ \pm}=\left(\frac{1}{4}+\frac{1}{4}\right) g_{ \pm}=\frac{1}{2} g_{ \pm}
$$

for $n_{1}=n_{2}=0$

and

$$
\left(n_{1}+n_{2}\right) \frac{1}{2} g_{ \pm}+\frac{1}{2} g_{ \pm}=g_{ \pm}
$$

for $n_{1}+n_{2}=1$ then the resonances occur at $g_{ \pm} \hbar \omega$ instead of at (1/2) $g_{ \pm}$, in which case, the resonances occur also at $\left[n_{3}+(1 / 2)\right] g_{ \pm} \hbar \omega_{c}$. The multiple particle resonances are weaker than at (1).

In the case of a cluster of electrons with spin up as well as spin down pairs, spin is zero for each pair, $s=0$, while the long range order is similar to that of an antiferromagnet, there is an exchange interaction which enhances the $g$ value. The spin zero gives, $\frac{1}{2} g=\frac{l+\frac{1}{2}}{2 l+1}=\frac{1}{2}$ so that $g=1$ and the energy levels occur at $\left(n+\frac{1}{2}\right) \frac{1}{2}$ which gives $1 / 4$ for $n=0$. The exchange enhancement changes this $1 / 4$ charge to the value, $\frac{1}{4}(g+\delta g) \cong \frac{1}{3.9}$ for $g=1$ and $\delta g=0.02564$ Therefore, in some of the samples, $1 / 4$ will appear as $1 / 3.9$. The value $\delta g$ can be understood in terms of exchange interaction and the effective charge becomes $e^{*} / e=1 / 3.9$. This effect will not occur in quenched samples and will be a characteristic of annealing. The spin clusters are not very 
stable.

\section{ELECTRON CLUSTERS IN GRAPHENE HALl EFFECT}

Recently, Bolotin et al. [19] have observed unusual fractions in the quantum Hall effect of graphene. From this study it is clear that the number of electrons in a cluster is a small number such as one or two or nine which are subject to exchange interaction which explains the deviations from the exact fractions. As the field moves away from the plateau there is clustering of electrons and hence there is a phase transition type phenomenon. The unusual features in the observation of Bolotin et al. are the sample dependent clusters, the experimental values of which are,

$$
\begin{gathered}
v=0.32 \pm 0.02=16 / 50, \\
v=0.68 \pm 0.05=34 / 50, \quad . . \\
v=0.30 \pm 0.02=3 / 10, \quad . . \\
v=0.46 \pm 0.02=23 / 50, \\
v=0.54 \pm 0.02=27 / 50 .
\end{gathered}
$$

We see that all of these sample-dependent fractions are correctly predicted and due to symmetries, we are able to arrange them in conjugate pairs. Pairing also helps in predicting the missing partners and looking for them. All of these denominators are even. Hence we take half of the denominator such as 25 which is $2 l+1=25$. Hence $l=12$ so that our expression,

$$
\frac{1}{2} g_{ \pm}=\frac{l+\frac{1}{2} \pm s}{2 l+1}=\frac{12+\frac{1}{2} \pm s}{25}=\frac{\frac{25}{2} \pm \frac{9}{2}}{25}=\frac{34}{50}, \frac{16}{50}=\frac{8}{25}
$$

This explains the two fractions given by (33). The calculated values agree with the measured values and identify the pairs correctly. A cluster of spin $9 / 2$ is formed in the sample due to defects on the graphene sheets. For $2 l+1=5$, $l=2$, we have,

$$
\frac{1}{2} g_{ \pm}=\frac{l+\frac{1}{2} \pm s}{2 l+1}=\frac{\frac{5}{2} \pm s}{5}=\frac{\frac{5}{2} \pm 1}{5}=\frac{3}{10}, \frac{7}{10}
$$

which explains (34) correctly and predicts $7 / 10$ as a conjugate of $3 / 10$. We thus see that clustering is the correct interpretation of the observation in graphene. The spin $s=1$ is correct for the electron pairs. The fraction $3 / 10$ is observed while $7 / 10$ is predicted. For $l=12$, we have,

$$
\frac{1}{2} g_{ \pm}=\frac{l+\frac{1}{2} \pm s}{2 l+1}=\frac{\frac{25}{2} \pm 1}{25}=\frac{27}{50}, \frac{23}{50}
$$

These values agree with experimentally observed values. This means that all of the experimentally found fractions agree with (i)principal fractions, (ii) resonances, (iii)two-particle states and (iv) clusters. The principal fractions are the property of single electrons while clusters are formed due to imperfections in the samples. There are phase transitions in going near the plateau [20]. The flux quantization depends on the spin [21]-[22]. The theory fits well with the Gell-Mann's theory of angular momentum [23]. It is found that Laughlin's theory is not related to the experimental data of quantum Hall effect. The bandshave been calculated along with the vibrational frequencies in defect models of graphene which show that the essential structure of our theory is correct [24]-[25]. The atomic clusters have been found in GaAs [26]. The $\mathrm{g}$ values also confirm that there is a zero value upon doping [27]. The basic theory of the quantized Hall effect has recently been explained without using two dimensionality as in graphite [28]-[29].

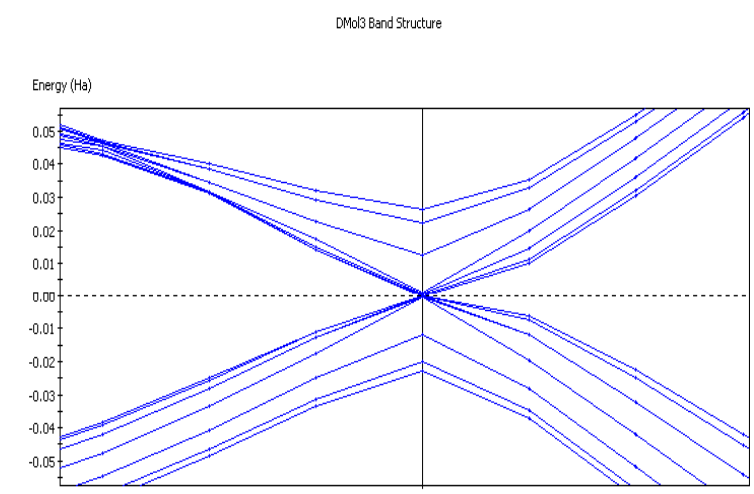

Fig. 3. The band structure of 12 layers of $\mathrm{AB}$ stacking of graphene

\section{CONCLUSIONS}

The quantum Hall effect of graphite is well explained by means of spin with Lande's formula replaced by a linear relation and suitable consideration of symmetries. The graphene data is also explained by the same theory. The states occur in pairs of left and right handed helicities associated with the $g$ values at high magnetic fields. The flux quantization becomes spin dependent and the fractional charge is determined by the angular momentum. Most of the fractions found in the data are explained by the single-particle theory. However, some of the fractions arise due to clustering in the samples. The samples of graphene tend to accumulate defects and hence electron clusters are found.

\section{REFERENCES}

[1] K. N. Shrivastava, "The quantum Hall effect: Interpretation of the experimental data," International Journal of Applied Physics and Mathematics, 2012, vol. 2, pp. 467-473.

[2] K. V. Klitzing, G. Dorda, and M. Pepper, "New method for high-accuracy determination of the fine structure constant," Phys. Rev. Lett., vol. 45, pp. 494-497, 11 August 1980.

[3] D. C. Tsui, H. L. Stormer, and A. C. Gossard, "Two-dimensional magnetotransport in the extreme quantum limit," Phys. Rev. Lett., vol. 48, pp. $1559-1562,1982$.

[4] R. B. Laughlin, "Anomalous quantum Hall effect: An incompressible quantum fluid with fractionally charged excitations," Phys. Rev. Lett., vol. 50, pp. 1395-1398, 1983.

[5] K. N. Shrivastava, "Six impossible things: Fractional charge from Laughlin's wave function," AIP Conference Proceedings, vol. 1325, pp.16-19, 2010. 
[6] C. Jiang, V. A. Shalygin, V. Y. Panevin, S. N. Danilov, M. M. Glazov, R. Yakimova, S. Lara-Avila, S. Kubatkin, and S. D. Ganichev, "Helicity dependent photocurrents in graphene layers excited by midinfrared radiation of a CO2 laser," Phys. Rev. B, vol. 84, pp. 125429 , 2011.

[7] K. N. Shrivastava, “Zero-energy state:The Lande's g value in quantum dots in AlGaAs," AIP Conference Proceedings, vol. 1325, pp. 90-94, 2010.

[8] P.-A. Huynh, F. Portier, H. le Sueur, G. Faini, U. Gennser, D. Mailly, F. Pierre, W. Wegscheider, and P. Roche, "Quantum coherence engineering in the integer quantum Hall regime," Phys. Rev. Lett., vol. 108 , pp. 256802-1-5, 2012.

[9] G. Granger, J. P. Eisenstein, and J. L. Reno, "Observation of chiral heat transport in the quantum Hall regime," Phys. Rev. Lett., vol. 102, pp. 086803-1-4, 2009.

[10] C. Altimiras, H. de Sueur, U. Gennser, A. Anthore, A. Cavanna, D. Mailly, and F. Pierre, "Chargeless heat transport in fractional quantum Hall regime,” Phys. Rev. Lett., vol. 109, pp. 026803, 2012.

[11] Y. Kopelevich, B. Raquet, M. Goiran et al.,"Searching for the fractional quantum Hall effect in graphite," Phys. Rev. Lett., vol. 103 pp. 116802-1-4, 2009.

[12] P. R. Wallace, "The band theory of graphite," Phys. Rev., vol. 71, pp. 622, 1947.

[13] S. Latil and L. Henrard, "Charge carriers in few-layer graphene films," Phys. Rev. Lett., vol. 97, pp. 036803-1-4, 2006.

[14] B. Partoens and F. M. Peeters, "Normal and Dirac fermions in grapheme multilayers: Tight binding description of the electronic structure," Phys. Rev. B, vol. 75, pp. 193402-1-3, 2007.

[15] E. J. Duplock, M.Scheffler, and P. J. D. Lindan, "Hallmark of perfect grapheme," Phys. Rev. Lett., vol. 92, pp. 225502-1-4, 2004.

[16] N. A. Zabidi, H. A. Kassim, and K. N. Shrivastava, "Energy band crossing points in multilayers of grapheme," AIP Conference Proceedings, vol. 1017, pp. 326-330, 2008.

[17] Y. Zhang, Y.-W. Tan, H. L. Stormer, and P. Kim, "Experimental observation of the quantum Hall effect and Berry's phase in grapheme," Nature, vol. 438, pp. 201-204, 2005.

[18] Y. Zhang, Z. Jiang, J. P. Small et al., "Landau-level splitting in grapheme in high magnetic fields", Phys. Rev. Lett., vol. 96, pp. 136806, 2006

[19] K. I.. Bolotin, F. Ghahari, M. D. Shulman, H. L. Stormer, and P. Kim, "Observation of fractional quantum Hall effect," Nature, vol. 462, pp. 196-199, 2009

[20] K. N.Shrivastava, "Plateau-to-plateau phase transition in quantum Hall effect," AIP Conference Proceedings, vol. 1250, pp. 27-30, 2010.

[21] K. N. Shrivastava, Proc. Conf. Honor C. N. Yang's $85^{\text {th }}$ Birthday, Statistical Physics, Hign Energy, Condensed matter and Mathematical Physics, World Scientific Pub. Co. Singapore, pp. 526, 2008.

[22] K. N. Shrivastava, "Byers and Yang's theorem on flux quantization," AIP Conference Proceedings, vol. 1250, pp. 261-264, 2010.

[23] K. N. Shrivastava, Proc. Conf. Honor Murray Gell-Mann's $80^{\text {th }}$ Birthday, quantum mechanics, elementary particles, quantum cosmology and complexity, World Sci. Pub. Co. Singapore, pp. 511-517, 2011
[24] N. A. Zabidi, H. A. Kassim, and K. N. Shrivastava, "DFT calculation of band structure of carbon chain pulled from grapheme," AIP Conference Proceedings, vol. 1250, pp. 241-244, 2010.

[25] N. A. Rosli, H. A. Kassim, and K. N. Shrivastava, "Graphene infrared spectroscopy: DFT vibrational frequencies," AIP Conference Proceedings, vol. 1250, pp. 269-272, 2010.

[26] V. R. Devi and K. N. Shrivastava, "DFT calculation of vibrational frequencies of GaAs and the Raman spectra," Spectrochimica Acta A, vol. 95, pp. 172-176, 2012.

[27] K. N. Shrivastava, "The Lande's g value in quantum dots in AlGaAs," International Journal of Nanoscience, World Sci. vol. 10, pp. 507-514 2011.

[28] K. N. Shrivastava, "The quantum Hall effect," AIP Conference Proceedings, vol. 1482, pp. 335-339, 2012.

[29] M. M. A. Ali and K. N. Shrivastava, "Symmetry properties of Laughlin's wave function and related states," AIP Conference Proceedings, vol. 1482, pp. 43-46, 2012.

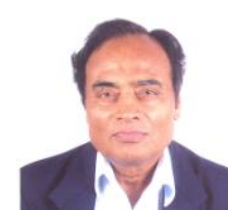

Keshav N. Shrivastava was born on July 11, 1943 and obtained his B.Sc. degree from the Agra University in 1961, M.Sc. degree from the University of Allahabad in 1963, Ph.D. from the Indian Institute of Technology Kanpur in 1966 and D.Sc. from Calcutta University in 1980.

He worked at the Clark University during 1966-68, at the Harvard University during the summer of 1968, at the Montana State University during 1968-69, and at the University of Nottingham during 1969-70. He was Associate Professor at the Himachal Pradesh University Simla, India during 1970-74. He worked at the University of California Santa Barbara during 1974-75 and at the State University Utrecht during 1975-76. He was Professor at the University of Hyderabad where he worked from 1978 till 2005. He worked in Tohoku University during 1999-2000. He visited the University of Zurich for short times during 1984, 1985, 1988 and $1999 . \mathrm{He}$ worked in the University of Houston in the summer of 1990 . He visited the Royal Institute of Technology Stockholm and the University of Uppsala during 1994. He visited the Technical University of Vienna,Austria in the summer of 1995. He visited the Princeton University and the University of Cincinnati during 2003. He was Professor in the University of Malaya Kuala Lumpur during 2005-2011. He is affiliated with the University of Hyderabad, India. He has worked in electron spin resonance, spin-phonon interaction, relaxation, Moessbauer effect, exchange interaction, superconductivity and the quantum Hall effect. He discovered the flux quantized energy levels in superconductors, the spin dependent flux and the correct theory of the quantum Hall effect. He has published more than 227 papers in journals and he is the author of the following books. (i) Superconductivity: Elementary Topics, World Scientific Singapore 2000. (ii) Introduction to Quantum Hall effect, Nova Sci. Pub N.Y. 2002 and (iii) The quantum Hall effect: Expressions, Nova Sci. N.Y. 2005.

Prof. Shrivastava is a Fellow of the Institute of Physics London, Fellow of the National Academy of Sciences India and member of the American Physical Society. 